\title{
Passively Mode-Locked Semiconductor Lasers and Their Applications
}

\author{
Sylwester Latkowski, Student Member, Frederic Surre, Member, IEEE, Pascal Landais, Member, IEEE \\ Research Institute on Networks and Communication Engineering, Dublin City University \\ Glasnevin, Dublin 9, Ireland \\ Tel: 353(01) 7008044,Fax: 353(01) 7005508,e-mail: landiasp@eeng.dcu.ie
}

\begin{abstract}
In this paper we present some characterizations of passively mode-locked semiconductor lasers. These lasers are multimode and they exhibit a modulation of their output power even though they are DC-biased. The modulation frequency corresponds to their free-spectral range. We demonstrate their potential: for generation of ultra-fast modulation for $\mathrm{THz}$ wave generation at room temperature without any direct modulations applied, in the radio frequency range they synchronise their modulation to the bit-rate of an incoming signal used for clock extraction. Therefore these devices can be used for all-optical clock recovery.
\end{abstract}

Keywords: multimode laser, passively mode-locking, radio-frequency, clock recovery, THz.

\section{INTRODUCTION}

Even though passively mode-locked (PML) lasers are DC biased, they exhibit a periodic variation of their output emission. This implies that they are cheap to use as they do not require any direct or external modulations. There are several types of configurations for edge emitting PML lasers:

- In 2001, Sato demonstrated pulse generation at $100 \mathrm{GHz}$ using a $408 \mu \mathrm{m}$ multi-quantum-well FabryPerot laser [1] and more recently Duan using a $1.5 \mu \mathrm{m}$ InP Q-dot FP laser [2].

- The gain-coupled distributed feedback (DFB) laser is another alternative. It is composed of two sub DFB sections merged to a phase section. Both sub-sections have a specific corrugation step and thus are spectrally detuned [3]. $40 \mathrm{GHz}$ self-modulation of this device has been demonstrated with its potential for clock-extraction [4]. The main drawback of these components is the complexity of their structure, which ultimately impacts their production cost.

- The distributed Bragg reflector (DBR) laser is also a solution. It is based on a large reflectivity spectrum of its Bragg mirror allowing at least two lasing FP modes in the longitudinal spectrum [5]. The potential for all-optical clock recovery has been demonstrated at $40 \mathrm{GHz}$ [6]. Key advantages of this device are that its reliable design leading to repeatable mode locking performance, low polarization dependence, a low power consumption and a reduced foot-print [6].

The modulation of the output is originated from the beating between the longitudinal modes co-existing in the cavity. It is found that the sum of the linewidth of each mode is larger than that of the beating signal. The down-converted signal benefits from a phase correlation between the lasing modes through the non-linear intra-cavity effects. Indeed, the four-wave-mixing (FWM) results in a modulation of the carrier population for detuning less than $100 \mathrm{GHz}$ [7]. It leads to a non-linear gain and refractive index modulation, affecting both the amplitude and the phase of the lasing modes. The phase of each mode is correlated to each other, resulting in a reduction of the phase noise, and thus the RF signal linewidth [5]. The formula (1) sets a criterion to identify the passive-modelocking in these devices:

$$
\Delta v_{\text {beating }}<<\sum \Delta v_{i}
$$

where $\Delta v_{\text {beating }}$ is the signal linewidth at the beating frequency and $\Delta v_{i}$ the linewidth of the $i^{\text {th }}$ optical mode. With the DBR laser presented above, a linewidth of less that $40 \mathrm{MHz}$ is achieved at $40 \mathrm{GHz}$. This results in a finesse of larger than 1000 required for all-optical clock recovery.

In this paper, we demonstrate the potential of this DBR laser to synchronise its $40 \mathrm{GHz}$ self-modulation to sub-harmonic signal at a bit-rate of 10 and $20 \mathrm{~Gb} / \mathrm{s}$. Furthermore based on FP laser with larger mode spacing, we demonstrate the potential of PML lasers for generation of $\mathrm{THz}\left(10^{12} \mathrm{~Hz}\right)$ signals at room temperature. The paper is organised as follow: Section 2 deals with the presentation of the PML DBR laser and the experimental set-up of all-optical synchronization, in Section 3, the THz PML laser is described with a demonstration of its performance of $\mathrm{THz}$ generation, finally in Section 4 conclusions are drawn. 


\section{OPTICAL SYNCHRONIZATION ON SUB-HARMONICS}

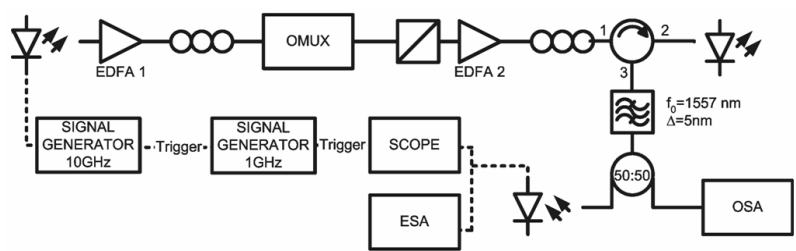

Figure 1. Set-up for optical synchronization on sub-harmonics of the PML DBR laser.

The laser under test is a bulk 3-section DBR laser. Each of the sections is electrically isolated from the other one by $3.71 \mathrm{k} \Omega$. The dimensions of InGaAsP bulk gain section are $790 \mu \mathrm{m}$ long $\times 0.6 \mu \mathrm{m}$ wide $\times 0.4 \mu \mathrm{m}$ thick exhibiting low polarization-sensitivity of modal gain and special single mode behaviour of the electric field. The phase section is $130 \mu \mathrm{m}$ long, with a waveguide width expanding linearly up to the $1.8 \mu \mathrm{m}$ width of the Bragg section. The short $200 \mu \mathrm{m}$ long Bragg section enables at least two longitudinal modes inside the $3 \mathrm{~dB}$ linewidth of the Bragg reflection spectrum. As a result, the DBR laser operates in either a multimode or a single mode regime depending on the bias condition. The extinction ratio of the output oscillation could be enhanced by adding to the described structure a saturable absorber section, not present in the device under test. The current in the gain section is labelled $I_{g}$, in the Bragg section $I_{B}$. The current in the phase section will always be set at $0 \mathrm{~mA}$ and will not be mentioned in the rest of the paper.

The experimental set-up is depicted in Fig. 1. A $10 \mathrm{GHz}$ pulse stream is generated by an externally modelocked laser. A $10 \mathrm{GHz}$ electrical signal is produced by a signal generator synchronized with second signal generator used to trigger a $50 \mathrm{GHz}$ sampling oscilloscope. The $10 \mathrm{GHz}$ signal stream is launched into an optical signal multiplexer, with an output at either $1 \times, 2 \times$, or $4 \times$ the initial $10 \mathrm{~Gb} / \mathrm{s}$ allowing rates at 10,20 , or $40 \mathrm{~Gb} / \mathrm{s}$. The format of the injected signal is return-to-zero, resulting in a strong power in the clock frequency and its harmonics. With a pseudo random data sequence more power should be required to achieve the same synchronisation performance as in a pulse series, but it will be doable as the data spectrum will contain a component at the clock frequency. The sequence injected corresponds to a series of pulses which is ideal for clock recovery as the power at the clock frequency is optimal. The input data signal is injected via a circulator into the DBR laser. The wavelength of the injected signal is $1567 \mathrm{~nm}$, at almost $10 \mathrm{~nm}$ above the DBR central wavelength. The $0 \mathrm{dBm}$ injected signal power is evaluated taking into account the coupling and injection losses. The signal output of the PML laser is transmitted from port 2 to 3 of the circulator and then analysed on either a $50 \mathrm{GHz}$ photodiode connected to a $50 \mathrm{GHz}$ electrical spectrum analyser or an optical spectrum analyser.

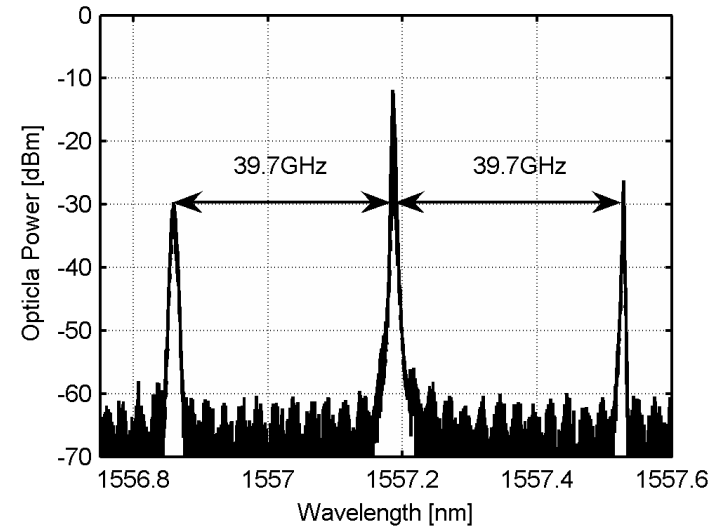

Figure 2. High resolution optical spectra produce by $S P-D B R$ device with no externally injected signal, biased at $I_{B}=1.75 \mathrm{~mA}$ and $I_{g}=181.49 \mathrm{~mA}$ and temperature controlled set at $T=298 \mathrm{~K}$.

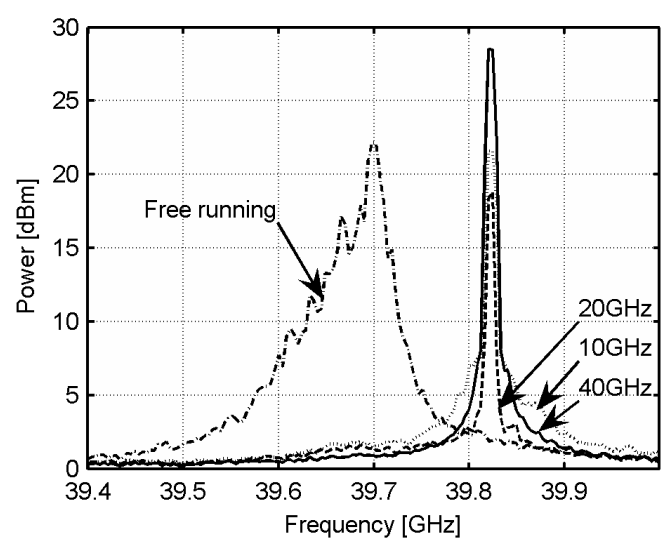

Figure 3. Electrical spectrum recorded for device biased at $181.83 \mathrm{~mA}$ on gain section and $1.75 \mathrm{~mA}$ on Bragg mirror with phase section unbiased and $3 \mathrm{dBm} 10 \mathrm{GHz}$ external optical signal injected.

With $I_{g}=181.83 \mathrm{~mA}$ and $I_{B}=1.75 \mathrm{~mA}$, the optical spectrum in free running condition, i.e. without data injection is recorded with an $8 \mathrm{fm}$ resolution optical spectrum analyser as depicted in Fig. 2. The longitudinal spectrum features three modes separated by $0.3 \mathrm{~nm}$, which corresponds to a $39.7 \mathrm{GHz}$ free spectral range (FSR) at $1550 \mathrm{~nm}$. The resolved linewidth is $224.57 \mathrm{MHz}, 74.133 \mathrm{MHz}$ and $81.521 \mathrm{MHz}$, for the mode 1,2 and 3 respectively. The side-mode-suppression ratio (SMSR) is approximately $17 \mathrm{~dB}$, confirming that the device is multimode. Under the same bias condition, the optical signal is launched into a $50 \mathrm{GHz}$ photodiode connected to a $40 \mathrm{GHz}$ electrical spectrum analyser. The electrical spectrum shown in Fig. 3 is recorded with a radio and a video bandwidth both set at $3 \mathrm{kHz}$. The signal is centred at $39.7 \mathrm{GHz}$, corresponding to the free-spectral range and its full width at half-maximum is $30.19 \mathrm{MHz}$. Applying equation (1), the sum of the optical linewidth is larger than the linewidth of the beating signal. The laser under test is passively mode-locked. 


\subsection{Results of all-optical synchronization on sub-harmonic bit-rate signals}

Bit-rates at 10,20 and $40 \mathrm{~Gb} / \mathrm{s}$ generated by temporally multiplexing a $10 \mathrm{GHz}$ pulse stream are injected into a $40 \mathrm{GHz}$ passively mode-locked DBR laser. The harmonics of the clock frequency embedded in the data stream spectrum synchronise the fundamental frequency of the PML laser. The response of the DBR laser is launched into the $50 \mathrm{GHz}$ photodiode and resolved with a $40 \mathrm{GHz}$ ESA. The performance of the synchronization is presented in Fig. 3. For 10, 20 and $40 \mathrm{~Gb} / \mathrm{s}$ injected signal, a shift from $39.7 \mathrm{GHz}$ to $39.82 \mathrm{GHz}$ is observed with a linewidth reduced from $30 \mathrm{MHz}$ to $13.8 \mathrm{MHz}$. The measures of the synchronized signal are actually limited by the resolution of the ESA. We demonstrated the synchronization of the $40 \mathrm{GHz}$ intrinsic modulation of this DBR laser on sub-harmonics at, 10 and $20 \mathrm{~Gb} / \mathrm{s}$, respectively and an all-optical clock recovery at $40 \mathrm{~Gb} / \mathrm{s}$. The performances achieved exhibit a large flexibility in terms of the bit-rate injected and also to the wavelength of the injected data stream.

\section{TERAHERTZ WAVE GENERATION}

As shown above, PML lasers can act as a reliable RF source with potential in clock recovery. Below a $100 \mathrm{GHz}$ FSR, the carrier density pulsation (CDP) is the four-wave-mixing (FWM) effect contributing to the PML process. Between $100 \mathrm{GHz}$ and $1 \mathrm{THz}$, the carrier heating $(\mathrm{CH})$ should be the dominant effect. Above $1 \mathrm{THz}$, both the CDP and the $\mathrm{CH}$ are weak and only the spectral hole burning (SHB) can produce mode beating. Thus it seems possible to generate a periodic modulation of the optical output of a multimode laser at frequency beyond $300 \mathrm{GHz}$, and thus in this case in the $\mathrm{THz}$ spectrum range, by exploiting mode beating due to material nonlinearities such as $\mathrm{CH}$ and $\mathrm{SHB}$.

The approach in order to assess this hypothesis is to use a multimode laser whose dominant modes wavelengths are separated by a few nanometers within the $3 \mathrm{~dB}$ gain spectrum. This will lead to a beating frequency of some hundreds of THz. One interesting solution from an application point of view, due to its cheap cost of production, is a Fabry-Perot laser. If the length of the cavity is appropriately selected, it should be possible to achieve a FSR from some hundreds of GHz to a few THz. Assuming a refractive index of 3.5 for the active layer, the length of the device will be $50 \mu \mathrm{m}$ and with associated mirror losses of $200 \mathrm{~cm}^{-1}$. The threshold current density over the active layer is of the order of $1.5 \cdot 10^{9} \mathrm{~A} / \mathrm{cm}^{2} . \mu \mathrm{m}$, well above the usual value of $5 \mathrm{kA} / \mathrm{cm}^{2} \cdot \mu \mathrm{m}$. Another approach, still based on a Fabry-Perot laser, is to implement a one dimension photonic band-gap in the laser cavity. The easiest way is to etch shallow grooves on the p-side of the laser junction perpendicular to the longitudinal axis of the device [8]. These grooves are not deep enough to reach the active layer, but they are deep enough to affect the effective refractive index experienced by the electromagnetic wave generated in the active layer. By etching these grooves at key positions it is possible to generate variation of the refractive index along the cavity and consequently to control the longitudinal spectrum. The FP resonance determines an initial spectral separation between consecutive modes, but the etched band-gap suppresses or enhances some specific FP modes. It is feasible therefore to select modes to achieve a given free-spectral range. In this case, we aim for a detuning in the THz range corresponding to a FSR between $2.5 \mathrm{~nm}$ and $1 \mathrm{~nm}$ at $1550 \mathrm{~nm}$.

The fact that the mode beating takes place within the laser cavity and not from an extended external cavity [9] or from the mixing of two laser sources [10] results in a more mechanically and thermally stable source apparatus. In our experiments, the laser is temperature controlled at $298 \mathrm{~K}$ and DC biased. It is a $350 \mu \mathrm{m}$ long multi-quantum well InAlGaAs Fabry-Perot (FP) laser. Its $2 \mu \mathrm{m}$ ridge waveguide provides a spatial single mode output. The grooves are $1 \mu \mathrm{m}$ deep and $2 \mu \mathrm{m}$ wide. The prototype device is characterized by a current threshold of $I_{t h}=19 \mathrm{~mA}$. Fig. 4 shows an optical spectrum at a bias current of $60 \mathrm{~mA}$. The resolution of the optical spectrum analyser is $0.05 \mathrm{~nm}$. The ripples are separated by $1 \mathrm{~nm}$ corresponding to a FSR of about $120 \mathrm{GHz}$ at $1550 \mathrm{~nm}$ and are due to the spurious Fabry-Perot modes of the $350 \mu \mathrm{m}$ cavity. The groove pattern designed on this device enhances a main mode at $1551.98 \mathrm{~nm}$ and a second mode at $1554.98 \mathrm{~nm}$ resulting with an approximate $39 \mathrm{~dB}$ SMSR, in these experimental conditions of bias current and temperature. As the laser spectrum exhibits two modes, it is theoretically possible to have a mode-beating between them, leading to the creation of a THz signal with the frequency $373 \mathrm{GHz}$. Furthermore the optical power associated with each mode will fluctuate with the same frequency as the beating signal.

Fig. 5 presents the $\mathrm{THz}$ spectrum for a bias current of $50 \mathrm{~mA}$ and a temperature of $298 \mathrm{~K}$ corresponding to the maximum $\mathrm{THz}$ signal detected. This measurement is carried out using a Fourier transform spectrometer equipped with a bolometer. The frequency of the $\mathrm{THz}$ peak is at $700 \mathrm{GHz}$ approximately, which corresponds, taking into account the error measurement and calibration to the second harmonic of the beating signal. 


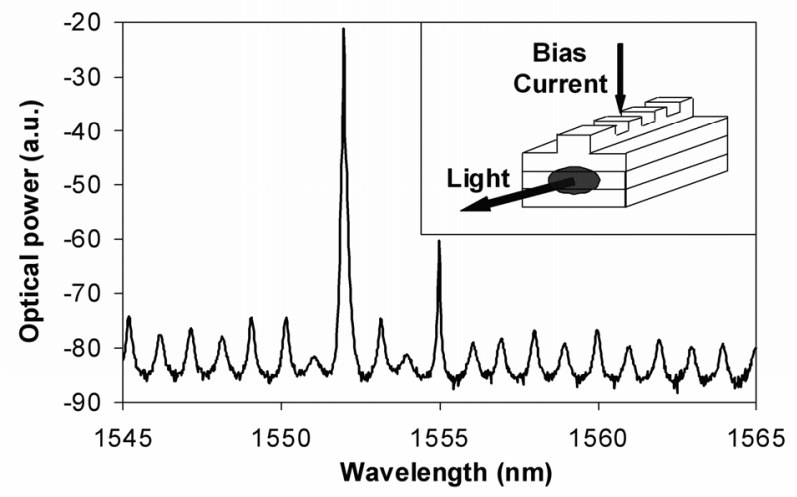

Figure 4. Optical spectrum of slotted laser for $I=60 \mathrm{~mA}$ and $T=298 \mathrm{~K}$

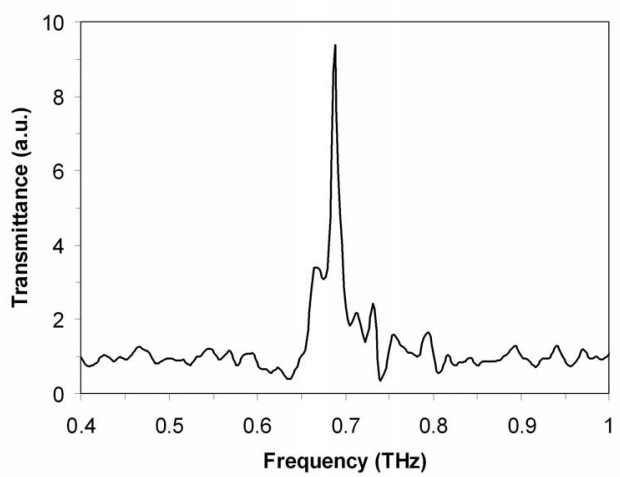

Figure 5. Terahertz spectrum for $I=50 \mathrm{~mA}$ and $T=298 \mathrm{~K}$.

The peak is approximately 10 times larger than the level of noise detected by this set-up. As the beating is enhanced by the phase correlation between optical modes, the linewidth of the THz signal, either first or second harmonic, is at most equal to the sum of the linewidth of both optical modes. These linewidths have been measured using a heterodyne technique at $30 \mathrm{MHz}$. Due to the spectrometer resolution of $1.8 \mathrm{GHz}$ and the small linewidth of the THz signal, it is not possible to find the exact value of the THz signal.

\section{CONCLUSIONS}

In this paper, we introduced two types of multimode lasers and demonstrated some applications of PML lasers. In telecommunication, all-optical clock recovery at $40 \mathrm{GHz}$ has been shown and furthermore $10 \mathrm{~Gb} / \mathrm{s}$ and $20 \mathrm{~Gb} / \mathrm{s}$ data signals were able to synchronise the DBR laser to its fundamental frequency. These experiments were carried out with a detuning between the DBR laser and the injected signal of almost $10 \mathrm{~nm}$. Using slotted FabryPerot laser with grooves implemented along the active layer, the resulting longitudinal spectrum has a freespectral range of $3 \mathrm{~nm}$ at $1554 \mathrm{~nm}$. The intra-cavity mode beating produces the generation of a $\mathrm{THz}$ signal at $298 \mathrm{~K}$. The use of FT-IR spectrometer has leaded to the measurement of a signal at $690 \mathrm{GHz}$ with a contrast ratio of approximately 10. This signal is the second harmonic of the FSR set by the groove pattern at $370 \mathrm{GHz}$.

\section{ACKNOWLEDGEMENTS}

This project is supported by SFI Research Frontier programme referenced 05/RFP/ENG0040.

The authors would like to thank Aragón Photonics for the use of their BOSA.

The authors are also grateful to Dr. S. Lynch, UCL, UK and Prof. W. Elsäßer, DUT, Germany for allowing THz measurements in their facilities.

\section{REFERENCES}

[1] K. Sato: $100 \mathrm{GHz}$ optical pulse generation using Fabry-Perot laser under continuous wave operation, Electronics Letters, vol. 37, pp. 763-764, June 2001.

[2] G.-H. Duan: Optical Signal Processing Using InP-Based Quantum-Dot Semiconductor Mode-Locked Lasers, in Proc. CLEO 2008, San-Jose, USA, May 2008, paper CMG3.

[3] M. Mohrle, et al.: Detuned grating multisection-RW-DFB lasers for high-speed optical signal processing, IEEE Journal of Selected Topics in Quantum Electronics, vol. 7, pp. 217-223, 2001.

[4] [13]W. Mao, et al:: All-optical clock recovery from RZ-format data by using a two-section gain-coupled DFB laser, Journal of Lightwave Technology, vol. 20, pp. 1705-1714, 2002.

[5] J. Renaudier, et al.: Phase correlation and linewidth reduction of $40 \mathrm{GHz}$ self-pulsation in distributed Bragg reflector semiconductor lasers, IEEE J. Quantum Electron. vol. 43, pp. 147-156, 2007.

[6] G-H. Duan, et al.: 40GHz all-optical clock recovery using polarization insensitive distributed Bragg reflector lasers, Lasers and Electro Optics, pp.1738-1740, 2003.

[7] G. Gray, et al.: Importance of self-induced carrier-density modulation in semiconductor lasers, IEEE Photonics Technology Letters, vol. 4, pp. 1216-1219, Nov. 1992.

[8] P. Landais, Patent No: S2005/0251 (Ireland, 26 April 2005).

[9] S. Hoffmann, et al: : Bandwidth limitations of two-colour diode lasers for direct terahertz emission, Electronics Letters, vol. 42, pp. 696-697, June 2006.

[10] S. M. Duffy, et al.: Accurate modeling of dual dipole and slot elements used with photomixers for coherent terahertz output power, IEEE Transactions on Microwave Theory and Techniques, vol. 49, pp. 1032-1038 June 2001. 